
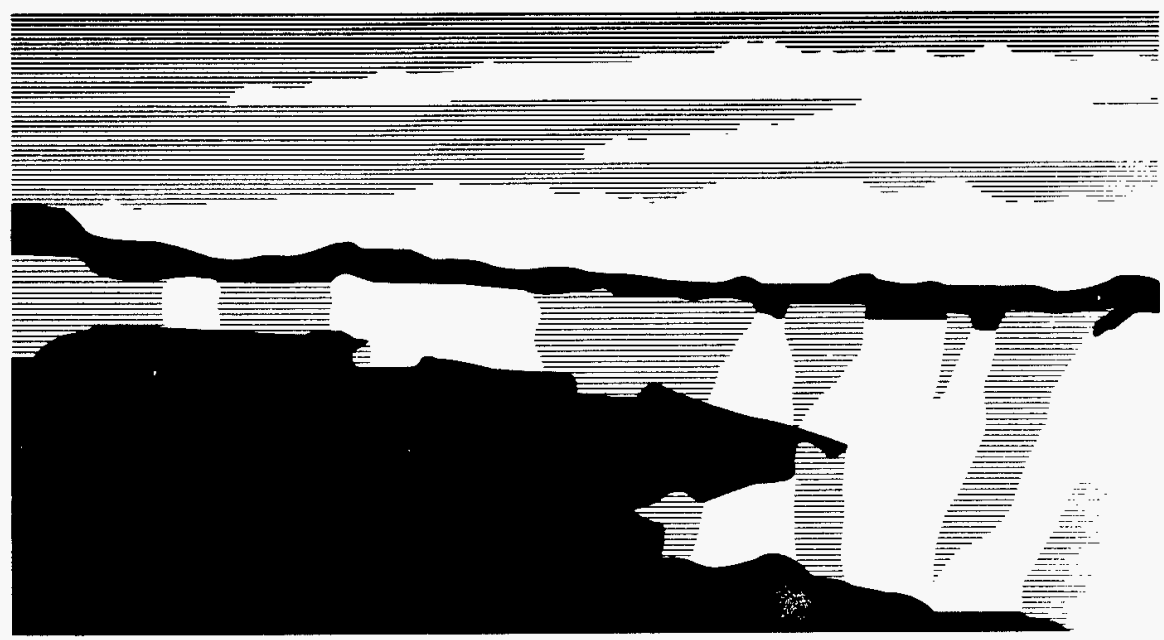

Los Alamos National Laboratory, an affirmative action/equal opportunity employer, is operated by the University of California for the U.S. Department of Energy under contract W-7405-ENG-36. By acceptance of this article, the publisher recognizes that the U.S. Government retains a nonexclusive, royalty-free license to publish or reproduce the published form of this contribution, or to allow others to do so, for U.S. Government purposes. The Los Alamos National Laboratory requests that the publisher identify this article as work performed under the auspices of the U.S. Department of Energy. 


\section{DISCLAIMER}

This report was prepared as an account of work sponsored by an agency of the United States Government. Neither the United States Government nor any agency thereof, nor any of their employees, makes any warranty, express or implied, or assumes any legal liability or responsibility for the accuracy, completeness, or usefulness of any information, apparatus, product, or process disclosed, or represents that its use would not infringe privately owned rights. Reference herein to any specific commercial product, process, or service by trade name, trademark, manufacturer, or otherwise does not necessarily constitute or imply its endorsement, recommendation, or favoring by the United States Government or any agency thereof. The views and opinions of authors expressed herein do not necessarily state or reflect those of the United States Government or.any agency thereof. 


\title{
LSND Neutrino Oscillation Results and Implications
}

\author{
William C. Louis, representing the LSND Collaboration [1] \\ Los Alamos National Laboratory, Los Alamos, NM 87545, USA
}

\begin{abstract}
The LSND experiment at Los Alamos has conducted searches for $\bar{\nu}_{\mu} \rightarrow \bar{\nu}_{e}$ oscillations using $\bar{\nu}_{\mu}$ from $\mu^{+}$decay at rest and for $\nu_{\mu} \rightarrow \nu_{e}$ oscillations using $\nu_{\mu}$ from $\pi^{+}$ decay in flight. For the $\bar{\nu}_{\mu} \rightarrow \bar{\nu}_{e}$ search, a total excess of $51.8_{-16.9}^{+18.7} \pm 8.0$ events is observed with $e^{+}$energy between 20 and $60 \mathrm{MeV}$, while for the $\nu_{\mu} \rightarrow \nu_{e}$ search, a total excess of $18.1 \pm 6.6 \pm 4.0$ events is observed with $e^{-}$energy between 60 and $200 \mathrm{MeV}$. If attributed to neutrino oscillations, these excesses correspond to oscillation probabilities (averaged over the experimental energies and spatial acceptances) of $(0.31 \pm 0.12 \pm 0.05) \%$ and $(0.26 \pm 0.10 \pm 0.05) \%$, respectively. For the future, the BooNE experiment at Fermilab could prove that neutrino oscillations occur and make precision measurements of $\Delta m^{2}$ and $\sin ^{2} 2 \theta$.
\end{abstract}

\section{Introduction}

One of the only ways to probe small neutrino masses is to search for neutrino oscillations, where a neutrino of one type (e.g. $\bar{\nu}_{\mu}$ ) spontaneously transforms into a neutrino of another type (e.g. $\bar{\nu}_{e}$ ). For this phenomenon to occur, neutrinos must be massive and the apparent conservation law of lepton families must be violated. In 1995 the LSND experiment [1] published data showing candidate events that are consistent with $\bar{\nu}_{\mu} \rightarrow \bar{\nu}_{e}$ oscillations. [2] Additional data are reported here that provide stronger evidence for $\bar{\nu}_{\mu} \rightarrow \bar{\nu}_{e}$ oscillations [3] as well as evidence for $\nu_{\mu} \rightarrow \nu_{e}$ oscillations. [4] The two oscillation searches have completely different backgrounds and systematics from each other.

\section{Detector}

The Liquid Scintillator Neutrino Detector (LSND) experiment at Los Alamos [5] was designed to search with high sensitivity for $\bar{\nu}_{\mu} \rightarrow \bar{\nu}_{e}$ oscillations from $\mu^{+}$decay at rest. The LANSCE accelerator is a most intense source of low energy neutrinos due to its $1 \mathrm{~mA}$ proton intensity and $800 \mathrm{MeV}$ energy: The neutrino source is well understood because almost all neutrinos arise from $\pi^{+}$or $\mu^{+}$decay; $\pi^{-}$ and $\mu^{-}$are readily captured in the $\mathrm{Fe}$ of the shielding and $\mathrm{Cu}$ of the beam stop. [6] The production of kaons and heavier mesons is negligible at these energies. The $\bar{\nu}_{e}$ rate is calculated to be only $4 \times 10^{-1}$ relative to $\bar{\nu}_{\mu}$ in the $36<E_{\nu}<52.8 \mathrm{MeV}$ energy range, so that the observation of a significant $\bar{\nu}_{e}$ rate" would be evidence for $\bar{\nu}_{\mu} \rightarrow \bar{\nu}_{e}$ oscillations.

The LSND detector consists of an approximately cylindrical tank $8.3 \mathrm{~m}$ long by $5.7 \mathrm{~m}$ in diameter. The center of the detector is $30 \mathrm{~m}$ from the neutrino source. On the inside surface of the tank 
1220 8-inch Hamamatsu phototubes provide $25 \%$ photocathode coverage. The tank is filled with 167 metric tons of liquid scintillator consisting of mineral oil and $0.031 \mathrm{~g} / \mathrm{l}$ of b-PBD. This low scintillator . concentration allows the detection of both Čerenkov light and scintillation light and yields a relatively long attenuation length of more than $20 \mathrm{~m}$ for wavelengths greater than $400 \mathrm{~nm}$. [7] A typical 45 $\mathrm{MeV}$ electron created in the detector produces a total of $\sim 1500$ photoelectrons, of which $\sim 280$ photoelectrons are in the Cerenkov cone. The phototube time and pulse height signals are used to reconstruct the track with an average r.m.s. position resolution of $\sim 30 \mathrm{~cm}$, an angular resolution of $\sim 12$ degrees, and an energy resolution of $\sim 7 \%$. The Čerenkov cone for relativistic particles and the time distribution of the light, which is broader for non-relativistic particles, give excellent particle identification. Surrounding the detector is a veto shield [8] which tags cosmic ray muons going through the detector.

\section{$3 \bar{\nu}_{\mu} \rightarrow \bar{\nu}_{e}$ Oscillation Data}

The signature for a $\bar{\nu}_{e}$ interaction in the detector is the reaction $\bar{\nu}_{e} p \rightarrow e^{+} n$ followed by $n p \rightarrow d \gamma(2.2$ $\mathrm{MeV}$ ). A likelihood ratio, $\mathrm{R}$, is employed to determine whether a $\gamma$ is a $2.2 \mathrm{MeV}$ photon correlated with a positron or is from an accidental coincidence. $\mathrm{R}$ is the likelihood that the $\gamma$ is correlated, divided by the likelihood that it is accidental. As shown in Fig. 1, R depends on the number of hit phototubes for the $\gamma$, the reconstructed distance between the positron and the $\gamma$, and the relative time between the $\gamma$ and positron. Fig. 2 shows the $R$ distribution, beam on minus beam off, for events with positrons in the $36<E<60 \mathrm{MeV}$ energy range.

The dashed histogram is the result of the $\mathrm{R}$ fit for events without a recoil neutron, and the solid histogram is the total fit, including events with a neutron. After subtracting the neutrino background with a recoil neutron, as shown in Table 1 , there is a total excess of $51.8_{-16.9}^{+18.7} \pm 8.0$ events, which if due to neutrino oscillations corresponds to an oscillation probability of $(0.31 \pm 0.12 \pm 0.05) \%$.

Figure 3 shows the electron energy distribution, beam on minus beam off excess, for events (a) without a $\gamma$ requirement and (b) events with an associated $\gamma$ with $R>30$. For this latter requirement, the total $2.2 \mathrm{MeV} \gamma$ detection efficiency is $23 \%$ and the probability that an event has an accidental $\gamma$ in coincidence is $0.6 \%$. The dashed histogram shows the background from expected neutrino interactions. There are 22 events beam on in the $36<E<60 \mathrm{MeV}$ energy range and a total estimated background (beam off plus neutrino-induced background) of $4.6 \pm 0.6$ events. The probability that this excess is a

statistical fluctuation is $<10^{-7}$. The observed average value of $\cos \theta_{b}$, the angle between the neutrino direction and the reconstructed positron direction, is $0.20 \pm 0.13$, in agreement with the expected value of 0.16 for $\bar{\nu}_{e} p$ interactions. If the observed excess is due to neutrino oscillations, Fig. 4 shows the allowed region ( $90 \%$ and $99 \%$ likelihood regions) of $\sin ^{2} 2 \theta$ vs $\Delta m^{2}$ from a maximum likelihoor 

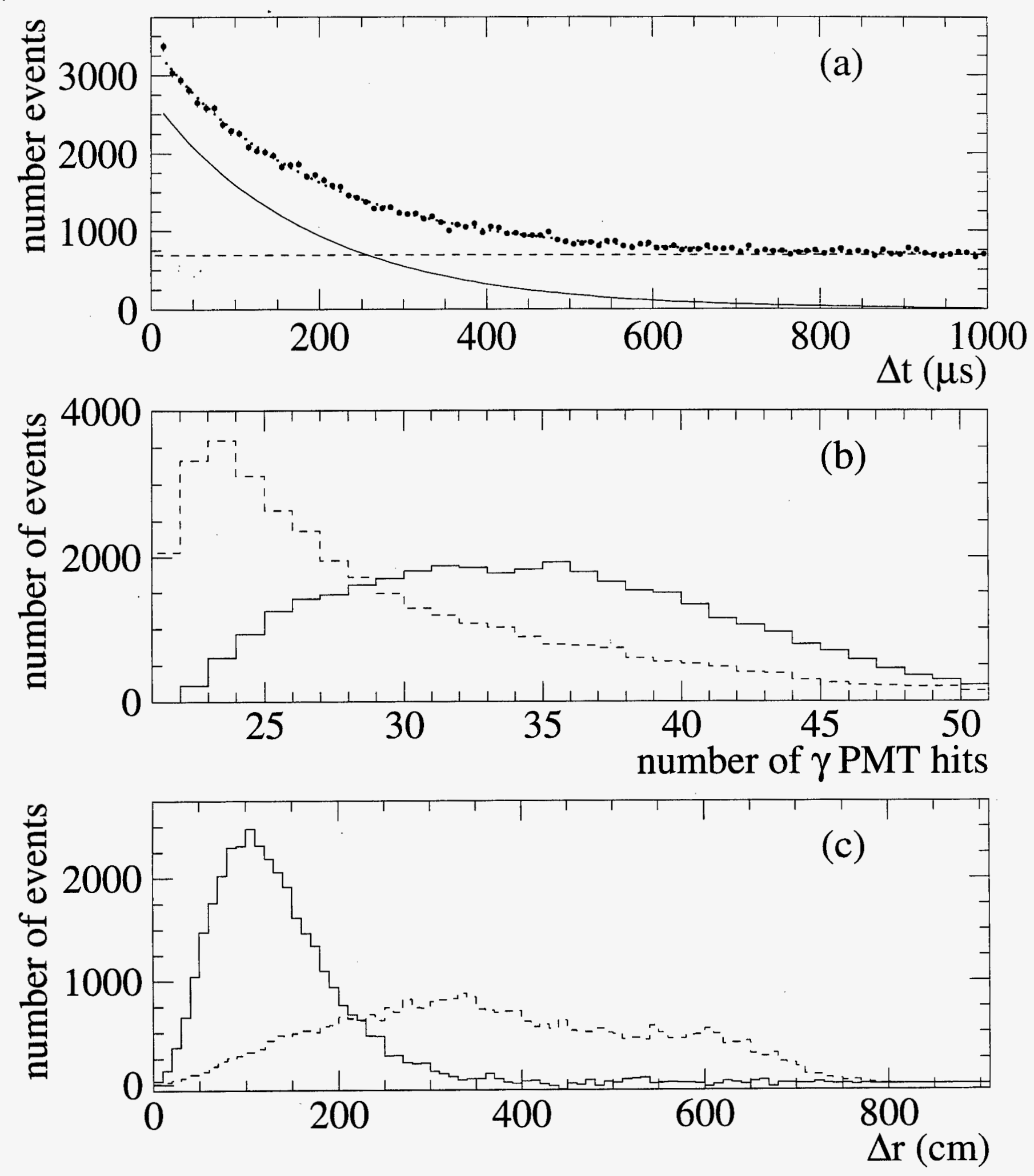

Figure 1: Associated $\gamma$ distributions in (a) time, (b) PMT hits, and (c) distance distributions for associated (solid) and accidental (dashed) $\gamma \mathbf{s}$. 


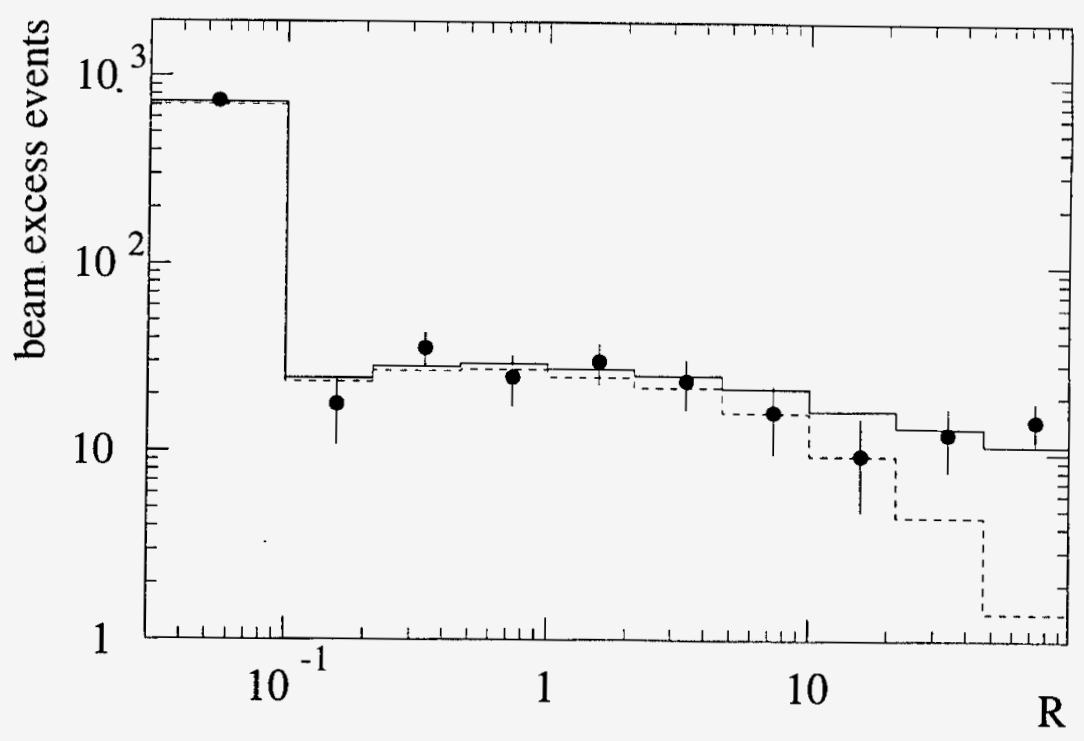

Figure 2: The $\mathrm{R}$ distribution, beam on minus beam off excess, for events that have energies in the range $20<E_{e}<60 \mathrm{MeV}$. The solid curve is the best fit to the data, while the dashed curve is the component of the fit with an uncorrelated $\gamma$.
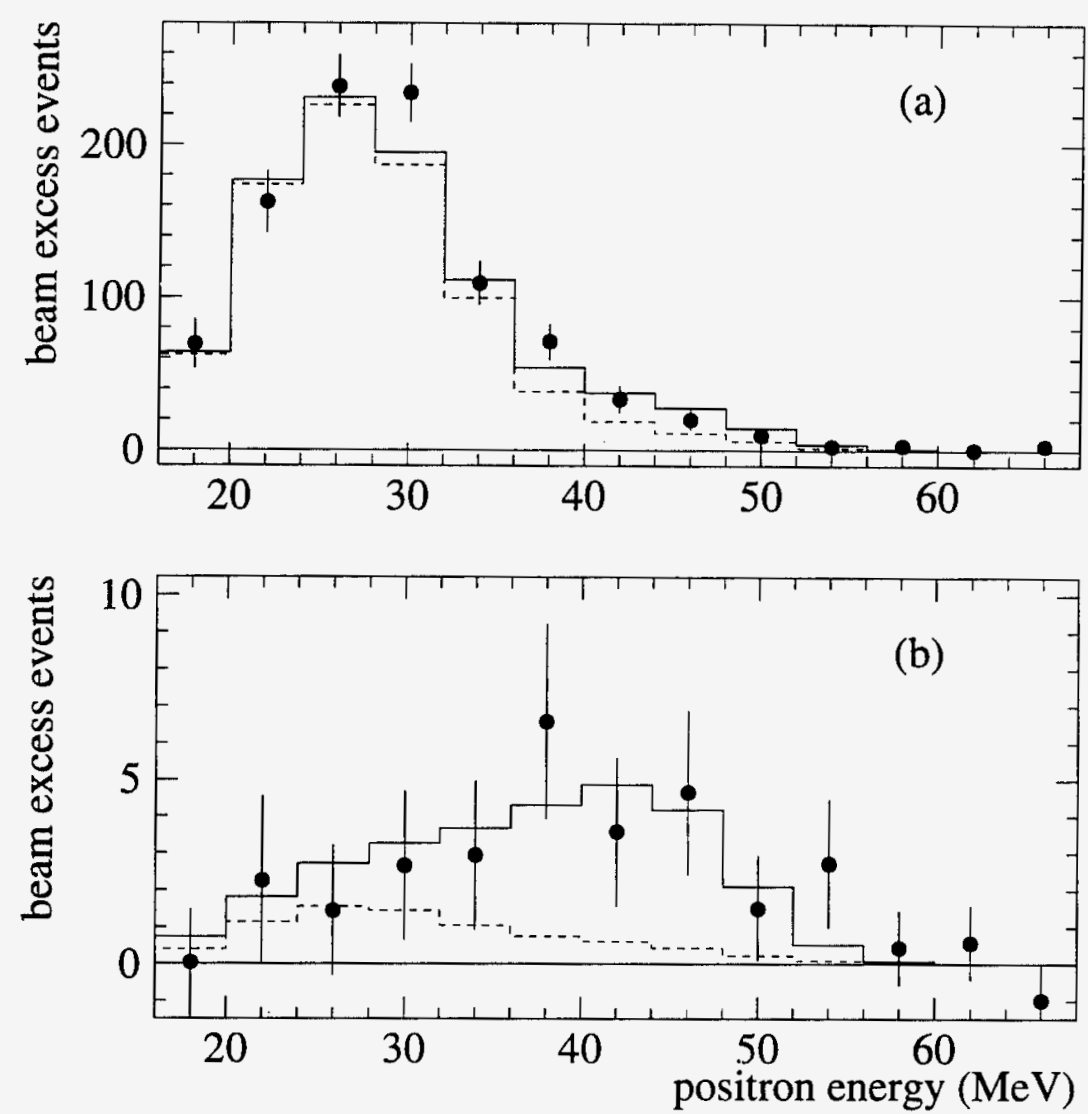

Figure 3: The energy distribution for events with (a) $R \geq 0$ and (b) $R>30$. Shown in the figure aw the beam-excess data, estimated neutrino background (dashed), and expected distribution for neutrin, oscillations at large $\Delta m^{2}$ plus estimated neutrino background (solid). 


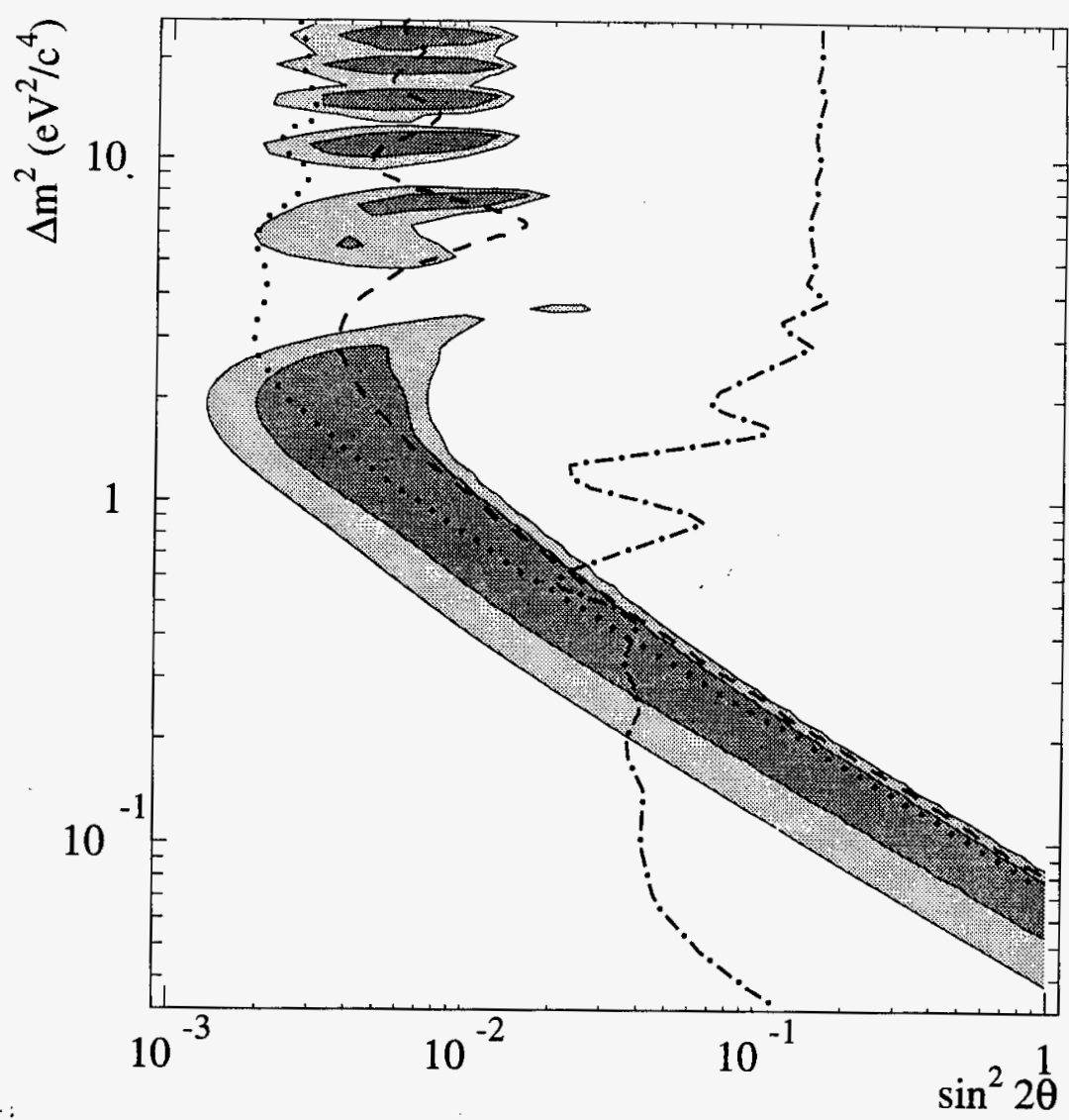

Figure 4: Plot of the LSND $\Delta m^{2}$ vs $\sin ^{2} 2 \theta$ favored regions. They correspond to $90 \%$ and $99 \%$ likelihood regions after the inclusion of the effects of systematic errors. Also shown are $90 \%$ C.L. limits from KARMEN at ISIS (dashed curve), E776 at BNL (dotted curve), and the Bugey reactor experiment (dot-dashed curve).

fit to the $L / E$ distribution of the entire data sample. Some of the allowed region is excluded by thr ongoing KARMEN experiment at ISIS, [9] the E776 experiment at BNL, [10] and the Bugey reactot experiment. [11]

Six months of additional data have been collected in 1996 and 1997. For this running periul the beam stop was reconfigured with the water target replaced by a close-packed high-z target fin testing tritium production. The muon decay-at-rest neutrino flux with this new configuration is $(m)$ $2 / 3$ of the neutrino flux with the old beam stop; however, the pion decay-in-flight neutrino flux $/ 1 .$. been reduced to $1 / 2$ of the original flux, so that the 1996 and 1997 data serve as a systematic chreh Preliminary results from 1996 and 1997 are given in Tables. 2 and 3. Table 2 shows the number : gold-plated events with $R>30$ from the entire 1993-1997 data sample, while Table 3 shows the tul., numbers of excess events and the corresponding oscillation probabilities from fits to the $\mathrm{R}$ distributin: for the running periods 1993-1995, 1996-1997, and 1993-1997. The preliminary oscillation probalıl!' : for the entire data sample is $(0.31 \pm 0.09 \pm 0.05) \%$ 


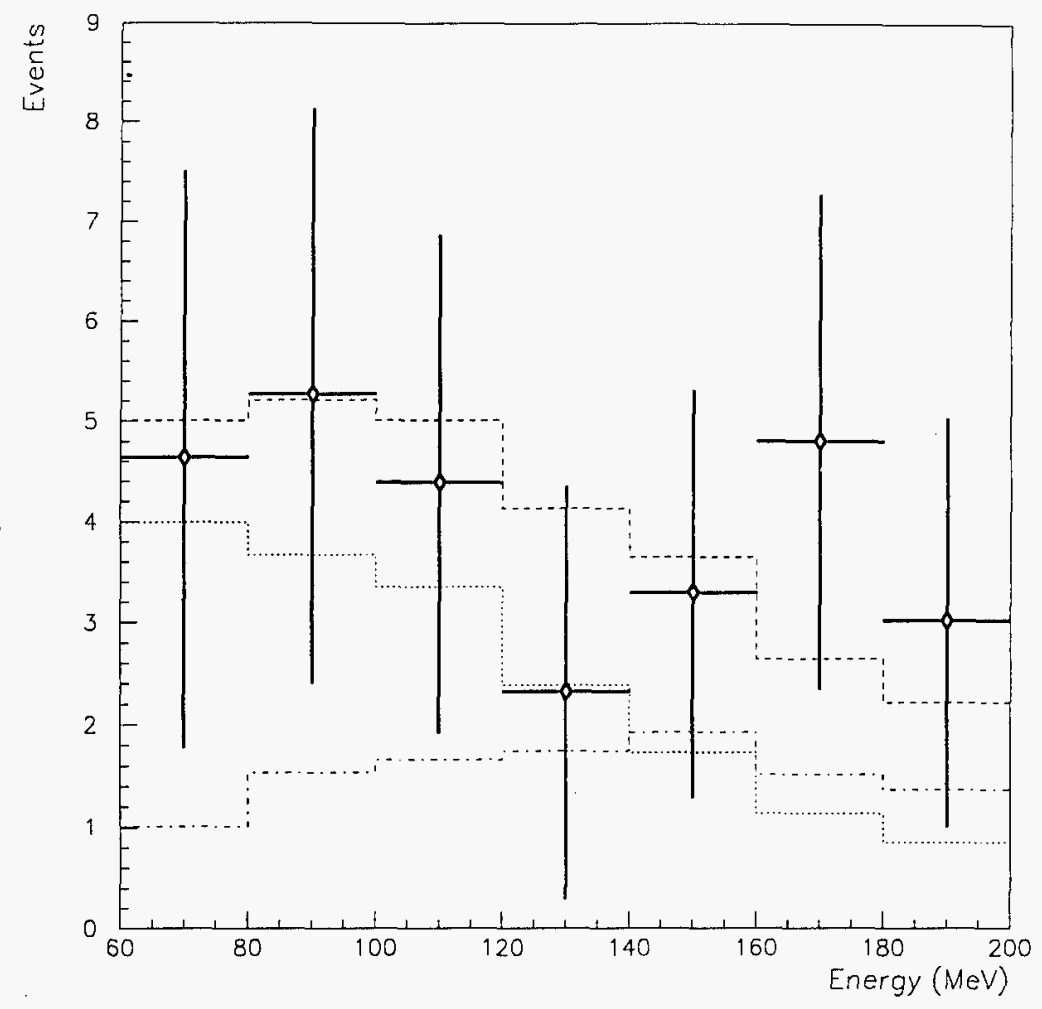

Figure 5: The energy distribution for the final beam-excess decay-in-flight events. The expectation for backgrounds (dot-dashed histogram), the oscillation signal for large values of $\Delta m^{2}$ (dotted histogram), and the sum of the two (dashed histogram) are shown.

\section{$4 \quad \nu_{\mu} \rightarrow \nu_{e}$ Oscillation Data}

The signature for $\nu_{\mu} \rightarrow \nu_{e}$ oscillations is an electron from the reaction $\nu_{e} C \rightarrow e^{-} X$ in the energy range $60<E_{e}<200 \mathrm{MeV}$. Using two independent analyses, [4] a total of 40 beam-related events and 175 beam-unrelated events are observed, corresponding to a beam on-off excess of $27.7 \pm 6.4$ events. The neutrino-induced backgrounds are dominated by $\mu^{+} \rightarrow e^{+} \bar{\nu}_{\mu} \nu_{e}$ and $\pi^{+} \rightarrow e^{+} \nu_{e}$ decays-in-flight in the beam-stop and are estimated to be $9.6 \pm 1.9$ events. Therefore, a total excess of $18.1 \pm 6.6 \pm 3.5$ events is observed above background. Fig. 5 shows the energy distribution of the beam on-off excess, along with the expectation for the backgrounds, for an oscillation signal at large $\Delta m^{2}$ and for the sum of the two. Fig. 6 shows the angular distribution for the beam on-off excess and the expectation from $\nu_{e} C \rightarrow e^{-} N$ scattering.

The excess events are consistent with $\nu_{\mu} \rightarrow \nu_{e}$ oscillations with an oscillation probability of $(0.26 \pm 0.10 \pm 0.05) \%$. A fit to the event distributions yields the allowed region in the $\left(\sin ^{2} 2 \theta, \Delta m^{2}\right)$ parameter space shown in Fig. 7 , which is consistent with the allowed region from the $\bar{\nu}_{\mu} \rightarrow \bar{\nu}_{e}$ search. These two searches have completely different backgrounds and systematic errors, and together they provide strong evidence that the observed event excesses are indeed due to neutrino oscillations. 


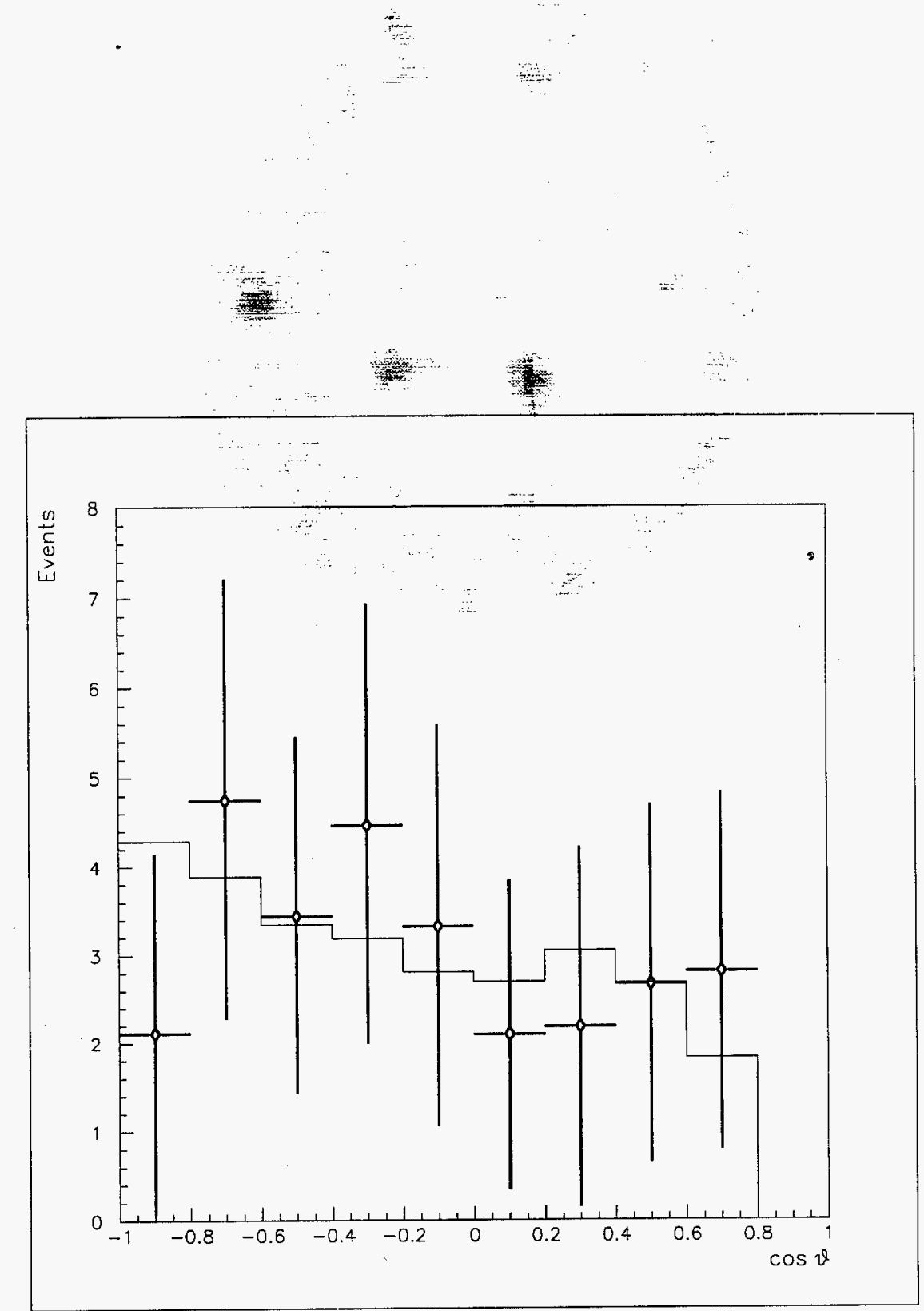

Figure 6: The angular distribution for the final beam-excess decay-in-flight events. The expectation for neutrino background and an oscillation signal at large values of $\Delta m^{2}$ (solid histogram) is shown. 


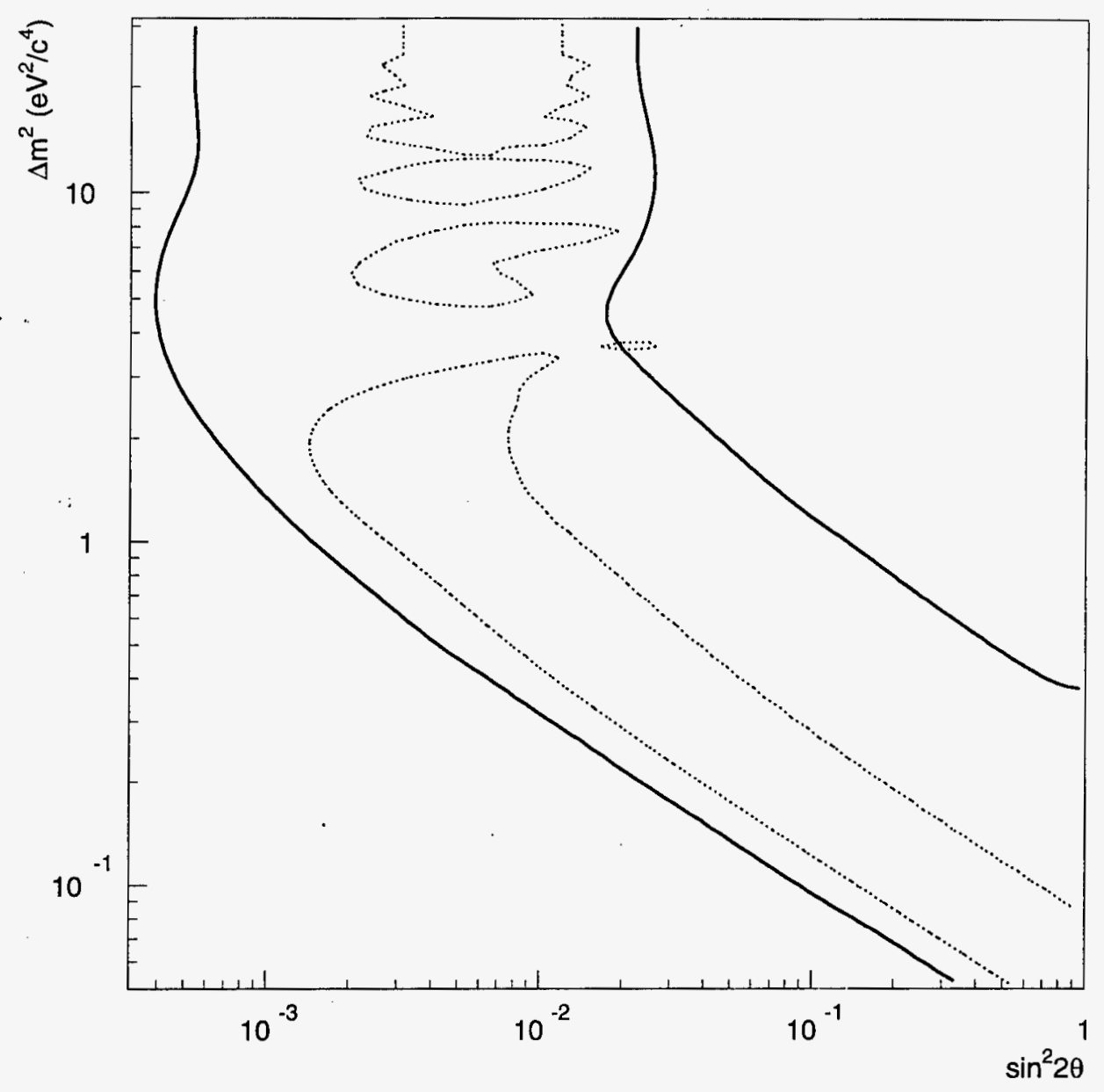

Figure 7: The $95 \%$ confidence region for $\nu_{\mu} \rightarrow \nu_{e}$ oscillations (solid curve) along with the farmmi regions for $\bar{\nu}_{\mu} \rightarrow \bar{\nu}_{e}$ oscillations (dotted curve). 


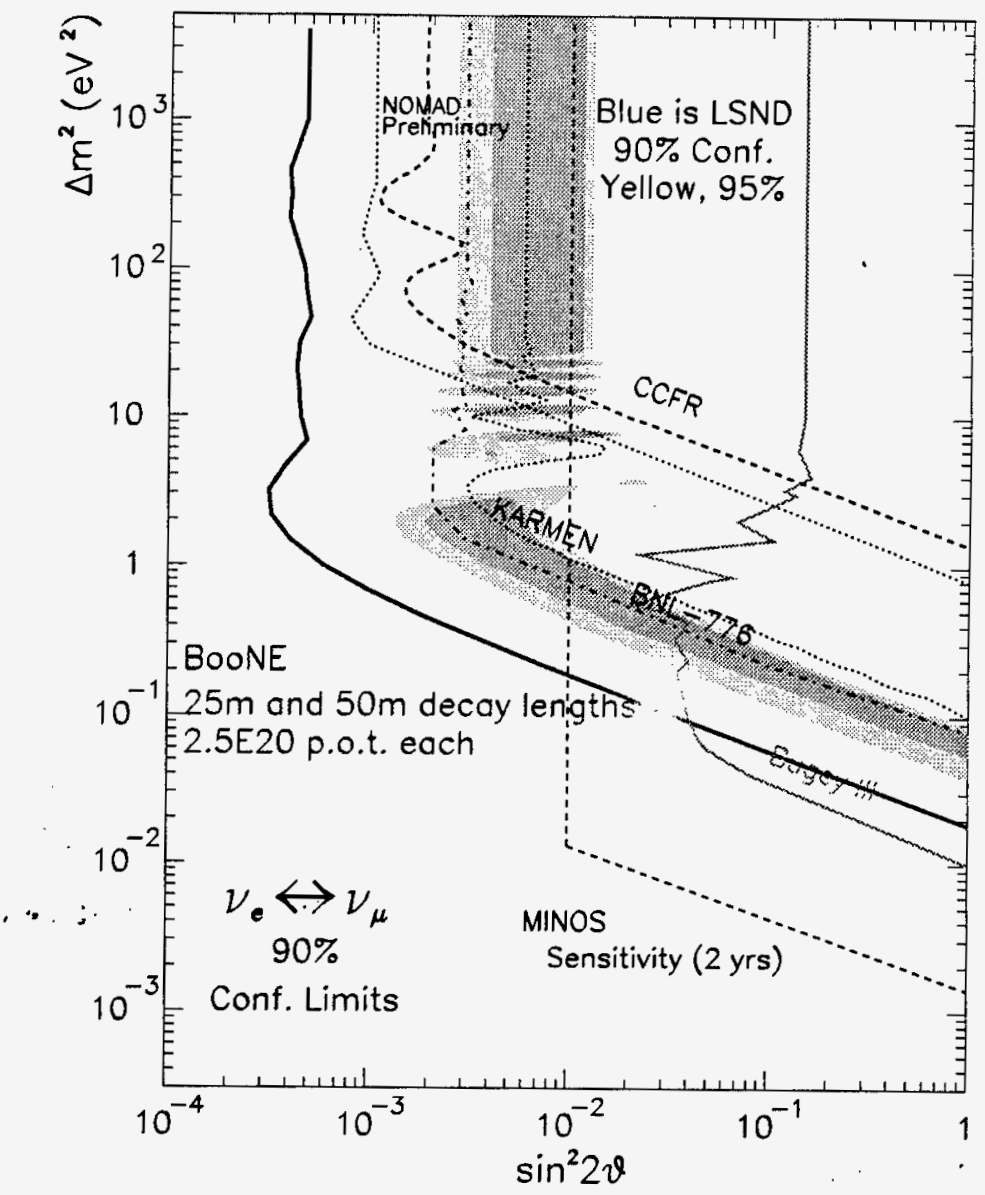

Figure 8: The 90\% C.L. limit expected from BooNE for $\nu_{\prime \prime} \rightarrow \nu_{e}$ appearance after one calendar year of running, including systematic errors, if LSND signal is not observed (solid line). Summary of results from past experiments and expectations for the future MINOS experiment are also shown.

\section{The BooNE Experiment at Fermilab}

Motivated by the LSND results, a Letter of Intent [12] has been submitted to Fermilab for building a Booster Neutrino Experiment (BooNE) to search for $\nu_{\mu} \rightarrow \nu_{e}$ oscillations and $\nu_{\mu}$ disappearance in the region of the LSND excess. Figs. 8 and 9 show the expected sensitivities for $\nu_{\mu} \rightarrow \nu_{e}$ appearance and $\nu_{\mu}$ disappearance after one calendar year of operation. Assuming that the LSND signal is verified, BooNE will be able to measure with precision the $\Delta m^{2}$ and $\sin ^{2} 2 \theta$ oscillation parameters and to search for CP violation in the lepton sector by comparing the $\nu_{\mu} \rightarrow \nu_{e}$ and $\bar{\nu}_{\mu} \rightarrow \bar{\nu}_{e}$ oscillation rates. Fig. 10 shows the number of events expected in 1 calendar year of running with BooNE for the low $\Delta m^{2}$ favored region of LSND (shaded). 


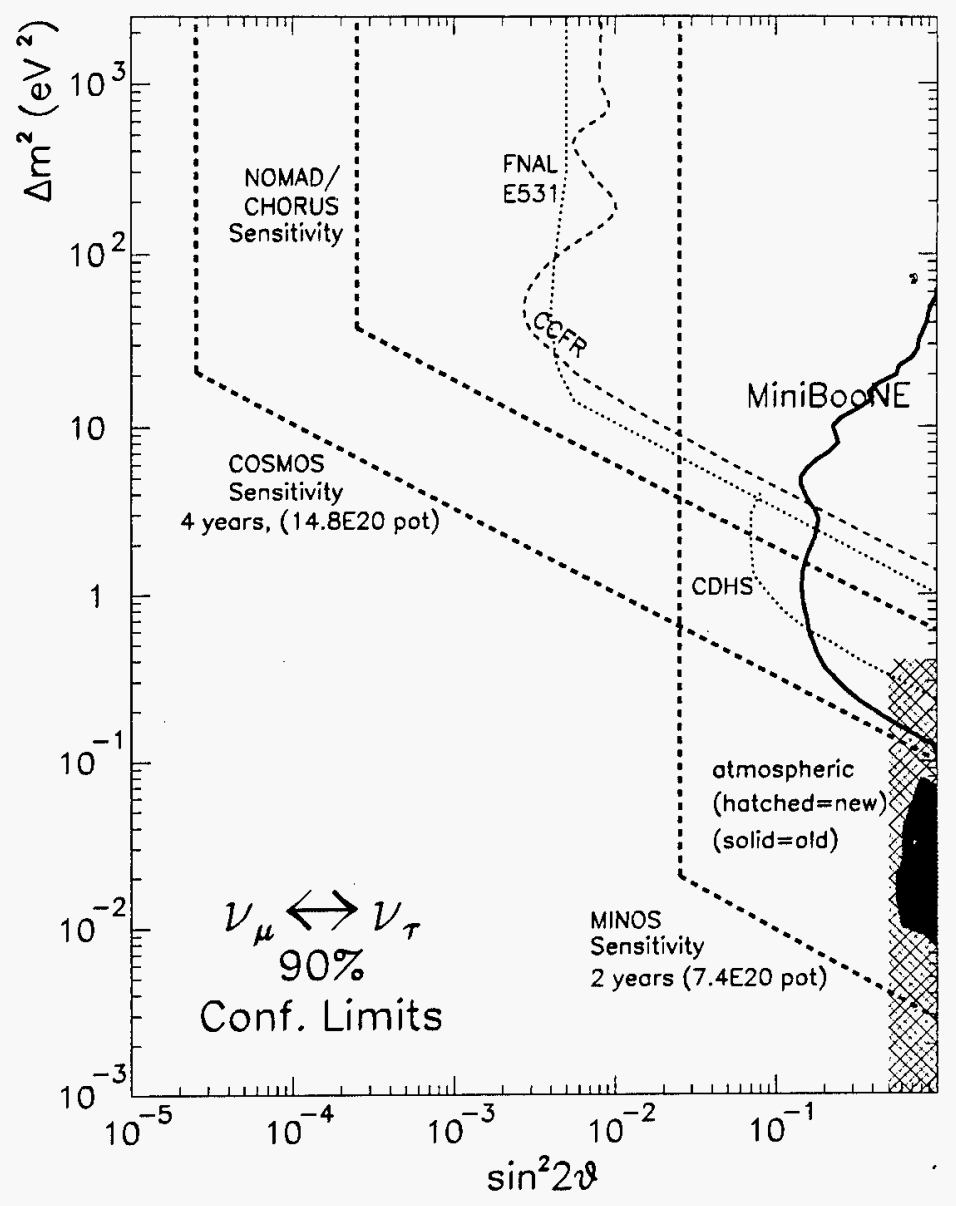

Figure 9: Summary of results from past experiments (narrow, dashed and dotted), future approved experiments (wide, dashed) and $90 \%$ C.L. limit expected for BooNE (solid) for $\nu_{\mu}$ disappearance after one calendar year of running at $0.5 \mathrm{~km}$. Solid region indicates the favored region for the atmospheric neutrino deficit from the Kamioka experiment. A result from Kamiokanda indicating no zenith angle dependence extends the favored region to higher $\Delta m^{2}$ as indicated by the hatched region. 


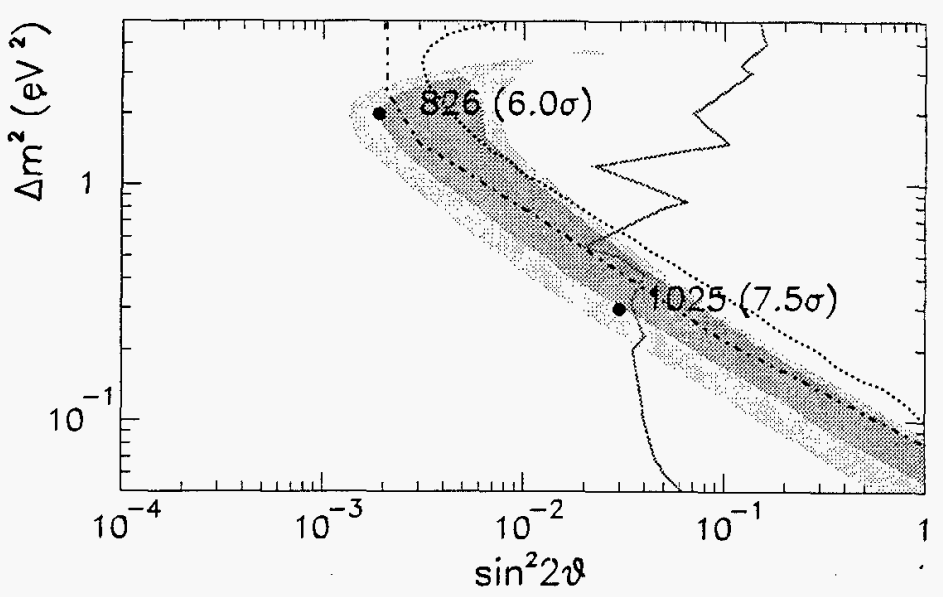

Figure 10: If the LSND signal is observed, this plot shows the number of events expected in 1 calendar year of running with BooNE for the low $\Delta m^{2}$ favored region of LSND (shaded). Lines indicate regions excluded by past experiments.

The BooNE experiment (phase 1) would begin taking data in 2001. By using phototubes and electronics from the LSND experiment, the BooNE Detector is relatively inexpensive, $\sim \$ 2 M$, and able to be constructed on a short time scale. The detector, as shown in Fig. 11, would consist of a spherical tank which is $12 \mathrm{~m}$ in diameter and covered on the inside by 1220 8-inch phototubes $(10 \%$ coverage) and filled with $750 \mathrm{t}$ of mineral oil, resulting in a $450 \mathrm{t}$ fiducial volume. The volume outside of the phototubes would serve as a veto shield for identifying particles both entering and leaving the detector. The detector would be located $500 \mathrm{~m}$ from the neutrino source. The second phase of the experiment would be to add an additional detector, that is identical to the first, at a distance of 1000 $\mathrm{m}$ from the neutrino source. The ratio of $\nu_{e} C \rightarrow e^{-N}$ events in the two detectors as a function of energy would allow the unambiguous determination of the oscillation parameters with much reduced systematic uncertainties.

The neutrino beam would be fed by the $8 \mathrm{GeV}$ proton Booster at FNAL and would service both phases of the experiment. The neutrino beam line would consist of a target followed by a focusing system and a $\sim 30 \mathrm{~m}$ long pion decay volume. The low energy, high intensity, and $1 \mu$ s time-structure of the Booster neutrino beam would be ideal for this experiment. The sensitivities shown in Figs. 8 and 9 assume the experiment receives $5 \mathrm{~Hz}$ of protons over one calendar year $\left(2 \times 10^{7} \mathrm{~s}\right)$. The cost if this beam line is expected to be $\sim \$ 4 \mathrm{M}$.

This Booster experiment is compatible with the Fermilab collider or the fixed-target MI programs. The FNAL Booster is capable of running at $15 \mathrm{~Hz}\left(5 \times 10^{12}\right.$ protons per pulse), or 30 Booster batche's per $2 \mathrm{~s}$ Main Injector Cycle. The antiproton stacking requires only 6 Booster batches at the start uf the Main Injector cycle. In principle, this means the BooNE beam line could receive $12 \mathrm{~Hz}$, well abor. 


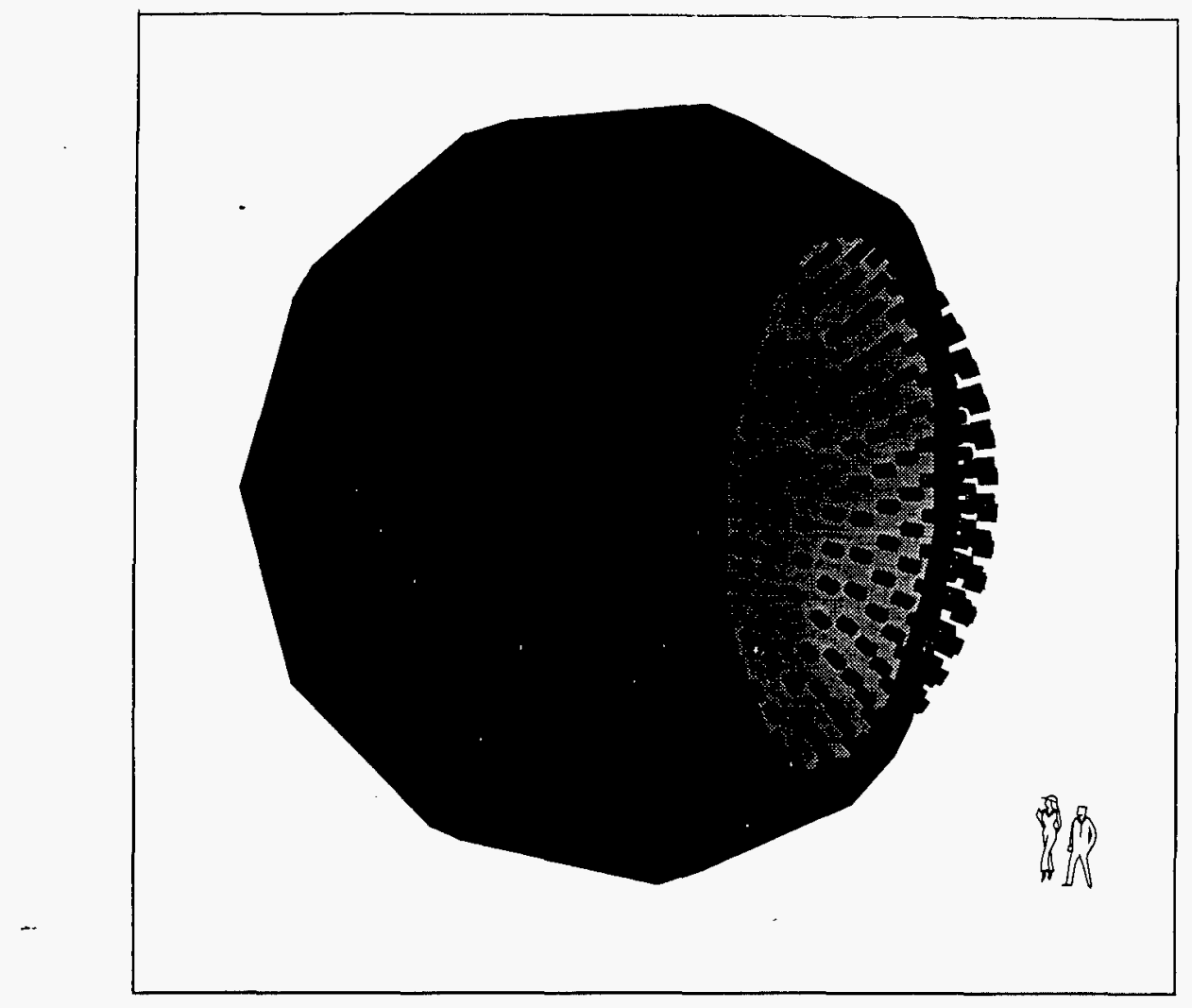

Figure 11: Schematic of the proposed BooNE detector.

the expectation on which the sensitivities are based.

The BooNE experiments represent an opportunity to resolve two outstanding neutrino oscillation questions on a short-time scale. Within the upcoming five years, no existing or approved experiments will be able tc address conclusively the LSND signal region. Thus BooNE represents an important and unique addition to the Fermilab program, and a formal proposal for this experiment will be submitted to the Fermilab Physics Advisory Committee in December of 1997.

\section{Conclusion}

In summary, the LSND experiment observes excesses of events for both the $\bar{\nu}_{\mu} \rightarrow \bar{\nu}_{e}$ and $\nu_{\mu} \rightarrow \nu_{e}$ oscillation searches, corresponding to oscillation probabilities of $(0.31 \pm 0.12 \pm 0.05) \%$ and $(0.26 \pm$ $0.10 \pm 0.05) \%$, respectively. These two searches have completely different backgrounds and systematics and together provide strong evidence for neutrino oscillatio in the range $0.2<\Delta m^{2}<2.0 \mathrm{eV}^{2}$. This implies that at least one neutrino has a mass greater than $0.4 \mathrm{eV}$. For the future, the BooNE experiment at Fermilab is capable of making precision measurements of the $\Delta m^{2}$ a $\sin ^{2} \cdot 2 \theta$ oscillation parameters. If neutrino oscillations have in fact been observed, then the minimal standard model would need to be modified and neutrinos would have mass sufficient to influence cosmology and the evolution of the universe. 


\section{* References}

[1] The LSND Collaboration consists of the following people and institutions: E. D. Church, K. McIlhany, I. Stancu, W. Strossman, G.J. VanDalen (Univ. of California, Riverside); W. Vernon (Univ. of California, San Diego); D.O. Caldwell, M. Gray, S. Yellin (Univ. of California, Santa Barbara); D. Smith, J, Waltz (Embry-Riddle Aeronautical Univ.); I. Cohen (Linfield College); R.L. Burman, J.B. Donahue, F.J. Federspiel, G.T. Garvey, W.C. Louis, G.B. Mills, V. Sandberg, R. Tayloe, D.H. White (Los Alamos National Laboratory);R.M. Gunasingha, R. Imlay, H.J. Kim, W. Metcalf, N. Wadia (Louisiana State Univ.): K. Johnston (Louisiana Tech Univ.); R. A. Reeder (Univ. of New Mexico); A. Fazely (Southern Univ); C. Athnassopoulos, L.B. Auerbach, R. Majkic, D. Works, Y. Xiao (Temple Univ.).

[2] C. Athanassopoulos et al., Phys. Rev. Lett. 75, 2650 (1995).

[3] C. Athanassopoulos et al., Phys. Rev. C 54, 2685 (1996); C. Athanassopoulos et al., Phys. Rev. Lett. 77, 3082 (1996).

[4] C. Athanassopoulos et al., LA-UR-97-1998, submitted to Phys. Rev. C.

[5] C. Athanassopoulos et al., Nucl. Instrum. Methods A 388, 149 (1997).

[6] R.L. Burman, M.E. Potter, and E.S. Smith, Nucl. Instrum. Methods A 291, 621 (1990); R.L. Burman, A.C. Dodd, and P. Plischke, Nucl. Instrum. Methods in Phys. Res. A 368, 416 (1996).

[7] R.A. Reeder et al., Nucl. Instrum. Methods A 334, 353 (1993).

[8] J.J. Napolitano et al., Nucl. Instrum. Methods A 274, 152 (1989).

[9] B. Bodmann et al., Phys. Lett. B 267, 321 (1991), B. Bodmann et al., Phys. Lett. B 280, 198 (1992), B. Zeitnitz et al., Prog. Part. Nucl. Phys. 32351 (1994).

[10] L. Borodovsky et al., Phys. Rev. Lett. 68, 274 (1992).

[11] B. Achkar et al., Nucl. Phys. B434, 503 (1995).

[12] E. Church et al., "A letter of intent for an experiment to measure $\nu_{\mu} \rightarrow \nu_{e}$ oscillations and $\nu_{\mu}$ disappearance at the Fermilab Booster", LA-UR-97-2120. 
Table 1: A list of all backgrounds with the expected number of background events in the $36<E_{e}<60$. $\mathrm{MeV}$ energy range for $R \geq 0$ and $R>30$. The neutrinos are from either $\pi$ and $\mu$ decay at rest $(\mathrm{DAR})$ or decay in flight (DIF). Also shown are the number of events expected for $100 \% \bar{\nu}_{\mu} \rightarrow \bar{\nu}_{e}$ transmutation.

\begin{tabular}{|c|c|c|c|}
\hline Background & Source & $R \geq 0$ & $R>30$ \\
\hline Beam off & & $146.5 \pm 3.2$ & $2.52 \pm 0.42$ \\
\hline Beam neutrons & & $<0.7$ & $<0.1$ \\
\hline \multicolumn{4}{|l|}{ DAR } \\
\hline $\bar{\nu}_{e} p \rightarrow e^{+} n$ & $\mu^{-} \rightarrow e^{-} \nu_{\mu} \bar{\nu}_{e}$ & $4.8 \pm 1.0$ & $1.10 \pm 0.22$ \\
\hline \multicolumn{4}{|l|}{ DIF } \\
\hline $\bar{\nu}_{\mu} p \rightarrow \mu^{+} n$ & $\pi^{-} \rightarrow \mu^{-} \bar{\nu}_{\mu}$ & $2.7 \pm 1.3$ & $0.62 \pm 0.31$ \\
\hline $\bar{\nu}_{e} p \rightarrow e^{+} n$ & $\pi \rightarrow e \nu$ and $\mu \rightarrow e \nu \bar{\nu}$ & $0.1 \pm 0.1$ & 0 \\
\hline Total & & $7.6 \pm 1.6$ & $1.72 \pm 0.38$ \\
\hline \multicolumn{4}{|l|}{ DAR } \\
\hline$\nu_{e}{ }^{12} C \rightarrow e^{-12} N$ & $\mu^{+} \rightarrow e^{+} \bar{\nu}_{\mu} \nu_{e}$ & $20.1 \pm 4.0$ & $0.12 \pm 0.02$ \\
\hline$\nu_{e}{ }^{13} C \rightarrow e^{-13} N$ & $\mu^{+} \rightarrow e^{+} \bar{\nu}_{\mu} \nu_{e}$ & $22.5 \pm 4.5$ & $0.14 \pm 0.03$ \\
\hline$\nu e \rightarrow \nu e$ & $\mu^{+} \rightarrow e^{+} \bar{\nu}_{\mu} \nu_{e}$ & $12.0 \pm 1.2$ & $0.07 \pm 0.01$ \\
\hline$\nu_{e} \mathrm{C} \rightarrow e^{-} X$ & $\pi \rightarrow e \nu_{e}$ & $3.6 \pm 0.7$ & $0.02 \pm 0.01$ \\
\hline \multicolumn{4}{|l|}{ DIF } \\
\hline$\nu_{\mu} \mathrm{C} \rightarrow \mu^{-} X$ & $\pi^{+} \rightarrow \mu^{+} \nu_{\mu}$ & $8.1 \pm 4.0$ & $0.05 \pm 0.02$ \\
\hline$\nu e \rightarrow \nu e$ & $\pi \rightarrow \mu \nu_{\mu}$ & $1.5 \pm 0.3$ & $0.01 \pm 0.01$ \\
\hline$\nu_{\mu} \mathrm{C} \rightarrow \pi X$ & $\pi \rightarrow \mu \nu_{\mu}$ & $0.2 \pm 0.1$ & 0 \\
\hline$\nu_{e} \mathrm{C} \rightarrow e^{-} X$ & $\pi \rightarrow e \nu$ and $\mu \rightarrow e \nu \bar{\nu}$ & $0.6 \pm 0.1$ & 0 \\
\hline Total & & $68.6 \pm 7.4$ & $0.41 \pm 0.04$ \\
\hline Grand Total & & $222.7 \pm 8.2$ & $4.65 \pm 0.57$ \\
\hline $100 \%$ Transmutation & $\mu^{+} \rightarrow e^{+} \bar{\nu}_{\mu} \nu_{e}$ & $12500 \pm 1250$ & $2875 \pm 345$ \\
\hline
\end{tabular}

Table 2: Preliminary numbers of gold-plated events wth $R>30$ from the entire 1993-1997 data sample.

\begin{tabular}{|c|c|c|c|c|}
\hline$e^{+}$Energy & Events Beam On & Events Beam Off & $\nu$ Background & Total Excess \\
\hline $20<E_{e}<60 \mathrm{MeV}$ & 61 & $15.6 \pm 1.0$ & $11.5 \pm 1.5$ & $33.9 \pm 8.0$ \\
$36<E_{e}<60 \mathrm{MeV}$ & 29 & $5.2 \pm 0.6$ & $3.0 \pm 0.6$ & $20.8 \pm 5.4$ \\
\hline
\end{tabular}


Table 3: Preliminary numbers of excess events and the corresponding oscillation probabilities from fits to the R distributions for the running periods 1993-1995, 1996-1997, and 1993-1997.

\begin{tabular}{|c|c|c|c|c|}
\hline Data Sample & Fitted Excess & $\nu$ Background & Total Excess & Oscillation Probability \\
\hline $1993-1995$ & $63.5 \pm 20.0$ & $12.5 \pm 2.9$ & $51.0 \pm 20.2$ & $(0.31 \pm 0.12 \pm 0.05) \%$ \\
$1996-1997$ & $35.1 \pm 14.7$ & $4.8 \pm 1.1$ & $30.3 \pm 14.8$ & $(0.32 \pm 0.15 \pm 0.05) \%$ \\
$1993-1997$ & $100.1 \pm 23.4$ & $17.3 \pm 4.0$ & $82.8 \pm 23.7$ & $(0.31 \pm 0.09 \pm 0.05) \%$ \\
\hline
\end{tabular}




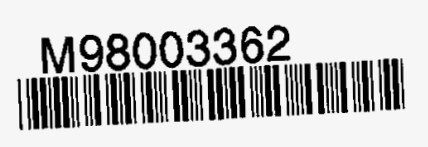

Report Number (14)LA-UR--97-4430
CONF-9709213

Publ. Date (11)

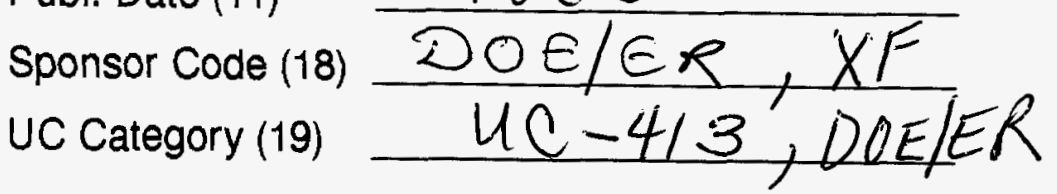

